\title{
Multimodal optimization for time-cost trade-off in construction projects using a novel hybrid method based on FA and PSO
}

\author{
Optimización multimodal para la compensación de tiempo-costo, en proyectos de construcción, \\ usando un nuevo método híbrido, basado en FA y PSO
}

Güllçă̆ Albayrak (Main and Corresponding Author)

Dept. of Civil Engineering, Eskisehir Osmangazi University

ESOGU Meselik Campus, 26480, Eskisehir, (Turkey)

galbayrak@ogu.edu.tr

illker Özdemir

Dept. of Civil Engineering, Eskisehir Osmangazi University

ESOGU Meselik Campus, 26480, Eskisehir, (Turkey)

iozdemir@ogu.edu.tr

Manuscript Code: 1051

Date of Acceptance/Reception: 01.08.2018/29.12.2017

DOI: $10.7764 / R D L C .17 .2 .304$

\begin{abstract}
Completion of the activities within optimal time and cost plays a significant role in construction projects. Recently, project managers have to decrease the total durations and costs of the projects more than before due to the competitive environment. Mostly, decision makers usually seek different alternatives which reduce time and cost. As well as being one of the most major topics of construction management, this problem called time-cost trade-off (TCTO) which is extremely difficult to solve with traditional mathematical methods. In recent years, metaheuristic algorithms are outstanding methods in this field due to their flexible and adaptable structure. This paper presents a new algorithm called F-PSO which consists of hybridizing Firefly Algorithm (FA) with Particle Swarm Optimization (PSO). In this method, the problem is modelled with various execution modes to select the optimal one for each activity. The applicability and validity of the proposed method is confirmed by performing 18 -activity project as a benchmark problem. Comparison of numerical results with different metaheuristic algorithms demonstrates the effectiveness and efficiency of F-PSO with regard to optimality of time and cost outcomes.
\end{abstract}

Key words: Hybrid algorithm, metaheuristic method, project planning, time-cost trade-off.

Resumen

La finalización de las actividades en un tiempo y costo óptimos desempeña un papel importante en los proyectos de construcción. Recientemente, los gerentes de proyecto tienen que disminuir la duración total y los costos de los proyectos más que antes debido al entorno competitivo. En general, quienes toman las decisiones generalmente buscan diferentes alternativas que reduzcan el tiempo y el costo. Además de ser uno de los temas más importantes de la gestión de la construcción, este problema se llama compensación - tiempo-costo (TCTO), que es extremadamente difícil de resolver con métodos matemáticos tradicionales. En los últimos años, los algoritmos metaheurísticos son métodos sobresalientes en este campo debido a su estructura flexible y adaptable. Este artículo presenta un nuevo algoritmo llamado F-PSO que consiste en hibridar el Algoritmo Firefly (FA) con la Optimización de Enjambre de Partículas (PSO). En este método, el problema se modela con varios modos de ejecución para seleccionar el óptimo para cada actividad. La aplicabilidad y validez del método propuesto se confirma realizando un proyecto de 18 actividades como un problema de referencia. La comparación de resultados numéricos con diferentes algoritmos metaheurísticos demuestra la efectividad y eficiencia de F-PSO con respecto a la optimización de los resultados de tiempo y costo.

Palabras clave: Algoritmo híbrido, método metaheurístico, planificación de proyectos, compensación de tiempo-costo.

Introduction

Generally, all activities are considered with normal durations in traditional project planning (Klerides \& Hadjiconstantinou, 2015). However, the decision makers sometimes have to complete the project earlier due to various reasons such as unforeseen delays, incentive contracts, imposed deadlines, contract commitments, overhead costs and pressure to move resources to other project. For this purpose, some of the activities must be accelerated to reduce the total project duration. This acceleration called crashing which can be achieved by additional resources or new technological changes, but they always cause increases in cost. Undoubtedly, the duration has a strong relation with the resource and they act each other inversely proportional. On the other hand, different time-cost relationships can occur depending on the cost components of the project. The most traditional form of time-cost relationship is expressed as linear. Linear time-cost relationship is a conventional deterministic model for defining the time-cost problems and numerous studies have been presented in the literature formerly. Kelley (1961), Fulkerson (1961), Siemens (1971), Salem \& Elmaghraby (1994), Hendrickson \& Au (1989), Tareghian \& Taheri (2006), and Chen \& Tsai (2011) put forward linear approaches for time-cost problems. 
The common point of these studies was that the relationship between time and cost of an activity was assumed as a linear function and the main purpose was to schedule the activities for minimizing the project cost, at that time (Vanhoucke \& Debels, 2007). Despite the fact that the linear approach is simply applicable as an exact method, it does not reflect the real-life problems precisely enough. Also exact methods can only optimize single-objective problems. However, if the optimization of time and cost is requested simultaneously, these methods fail. This optimization problem is known as time-cost trade-off (TCTO) problem in the literature (Hegazy, 1999). The concept of TCTO has been raised with the multi execution modes which include different time-cost alternatives for activities. This type of TCTO models called discrete optimization has combination of discrete points corresponding to each time-cost set of the activities. In project planning, the activities which have at least two modes are considered as multimodal optimization problems. The aim of ТСТО is to find the best alternative solution by providing the optimal total duration and cost of the project.

Our intent with this paper is to present the applicability of an alternative hybrid intelligent search method for solving the problem of the minimization of total project cost and duration, when discrete time-cost combinations are available on the activities of a project. This paper is different from the previous studies with respect to proposing a novel method for the solution. Moreover, presenting a Pareto front of results to decision makers gives them the opportunity to select the better solution due to project limitations and make the decision making procedure more flexible. The mathematical model of ТСТО is described considering multimodal and multi-objective optimization requirements. Also a novel hybrid algorithm (Firefly-Particle Swarm Optimization, abbr. F-PSO) is proposed to solve TCTO problem. F-PSO is based on PSO combining with FA approach for TCTO problems in construction projects. In this algorithm, PSO searches the solution space globally when FA focuses on finding optimal solutions. Thus, the advantages of both nature inspired algorithms are combined. Furthermore, the proposed F-PSO method is tested on 18-activity TCTO problem which is one of the most well-known problems derived from the literature. For demonstrating the performance of F-PSO, the obtained numerical solutions are compared with other studies conducted with different metaheuristic methods existing in the literature.

The remaining content of this paper is organized as follows: TCTO is introduced and the mathematical model is explained in the section of Description of the Problem. The related works which apply metaheuristic methods on TCTO are mentioned in the section of State of Art. In the section of Methodology, Firefly Algorithm (FA) and Particle Swarm Optimization (PSO) are summarized and F-PSO is presented. Also, application of F-PSO on the case example is given in the same section. In the section of Results, the computational results are discussed. Finally, conclusions and directions of future work are mentioned in the section of Conclusions.

Description of the Problem

ТСТО is a multi-objective optimization which aims to minimize both time and cost. The optimization brings the optimal time-cost sets which constitute Pareto front. Pareto front was first formulated by Pareto (1906) as the commonly accepted tool for evaluating potential solutions. Pareto front is a set of non-dominated solutions being chosen as optimal if no objective can be improved without sacrificing at least one other objective (Fan, You, \& Li, 2013). Thus, the decision maker can find the most appropriate time-cost set, which is undetectable by conventional methods, according to subjective preferences.

The mathematical model of TСТО problem given in the below is adapted from a previous study which is conducted by the first author of this paper (Albayrak, 2017). The mathematical model is described by Equations (1- 4) as constraints and Eq. (5) and (6) as objective functions. In Equations ( $1-6), c_{t}, t_{t}, c_{i j}$ and $x_{i j}$ represent total cost, total duration, cost of the $j^{\text {th }}$ mode for $i^{\text {th }}$ activity, assignment of the $j^{\text {th }}$ mode for $i^{\text {th }}$ activity respectively. $T, m n, n, T_{i j}$ and $T_{\max }$ represents starting time, mode alternatives, activity number, duration of $j^{\text {th }}$ mode of $i^{\text {th }}$ activity and maximum completion time respectively. According to the Eq. (1), the project starts at time 0 . Eq. (2) states that, the sum of the starting time of the last activity with duration should be less than or equal to the maximum completion time of the project. According to Eq. (3), the sum of the starting time of a predecessor activity and the duration of $j^{\text {th }}$ mode should be less than or equal to starting time of the successor activity. The precedence constraints must not be violated. The last constraint Eq. (4) expresses that only one mode must be assigned for each activity. Accordingly, $x_{i j}$ is a binary variable which takes the value of $0-1$.

Constraint functions:

$T_{1}=0$;

$T_{n}+\sum_{j=1}^{m_{n}} T_{n j} \cdot x_{n j} \leq T_{\max }$

$T_{a}+\sum_{j=1}^{m_{a}} T_{a j} \cdot x_{a j} \leq T_{b} ; a \rightarrow b$ for all predecessors $\mathrm{a}, \mathrm{b}=1, \ldots, \mathrm{n}$;

$\sum_{i=1}^{n} \sum_{j=1}^{m_{i}} x_{i j}=1$; 
Objective functions:

Min $c_{t}=\sum_{i=1}^{n} \sum_{j=1}^{m_{i}} c_{i j} x_{i j}$

$\operatorname{Min}_{t}=\left[T_{n}+\sum_{j=1}^{m_{n}}\left(T_{i j} \cdot x_{i j}\right)\right]$

First objective function Eq. (5) aims to minimize total project cost and second objective function Eq. (6) aims to minimize total project duration.

\section{State of the Art}

The time-cost trade-off problem has been extensively studied decades ago under the assumption of continuous timecost relationships. On the contrary, the literature for the case where the time-cost modes are defined at discrete points (representing distinct alternatives) is relatively recent, despite the fact that the discrete time-cost modes are considered to be a more realistic model of real projects. Undoubtedly, the problem has gained considerable attention. Because mathematical structure of the problem includes complicated formulation and, thus, it is very difficult to obtain optimal solutions efficiently. De, Dunne, Ghosh, \& Wells (1997) and also Deineko \& Woeginger (2001), showed that this problem belongs to NP-hard (non-deterministic polynomial time) problem. Due to unlikely existence of any polynomial algorithm to solve this problem optimally, the efforts have turned to finding the approximation and heuristics methods. The metaheuristic algorithms, which include a number of different methods, have an important role in this field because of their widespread application area than conventional methods such as critical path method and linear programming.

Li \& Love (1997), Feng, Liu, \& Burns (1997; 2000), Leu \& Yang (1999) and (Hegazy, 1999) are the initiative studies in the literature in terms of proposing metaheuristic method based on Genetic Algorithm (GA) to solve TCTO. Thereafter TCTO has been extensively studied and many different algorithms have been suggested by many researchers. New powerful metaheuristic algorithms, such as Simulated Annealing, Tabu Search, Ant Colony Algorithm (ACO), Particle Swarm Optimization and Shuffled Frog Leaping Algorithm, have contributed to further development of optimization. The large number of metaheuristics proposed to solve the problem, as well as the values to be decided for many parameters of a metaheuristic, impose their evaluation with regard to their time efficiency and the quality of the obtained solutions. The studies related to TCTO solving with metaheuristic algorithms are as follows mainly: Elbeltagi, Hegazy, \& Grierson (2005) presented five evolutionary-based optimization algorithms with comparison. Tareghian \& Taheri (2007) introduced the electromagnetic scatter search algorithm for discrete TCTO problem. Huang, Deng, \& Zhang (2008) and Ng \& Zhang (2008) proposed ACO. Eshtehardian, Afshar, \& Abbasnia (2009) presented a fuzzy multi-objective GA approach for uncertain environment conditions. Geem (2010) applied a Harmony Search (HS) algorithm to solve timecost trade-off problems. The objective is to minimize total cost, which consists of direct cost and time related indirect cost. Mokhtari, Baradaran Kazemzadeh, \& Salmasnia (2011) applied the ant colony system to the multimodal discrete TCTO problem with normal distribution of activity time values. Sonmez \& Bettemir (2012) proposed a new hybrid algorithm using Simulated Annealing, Quantum Based Simulated Annealing and GA. Cheng \& Tran (2014) proposed a novel approach by introducing their two-phase differential evolution model which was able to successfully reflect both time-cost effects and resource constraints. Prascevic \& Prascevic (2014) applied PSO method for solving time-cost optimization, and developed for that purpose an appropriate computer programme. Lee, Yi, Lee, \& Arditi (2015) used the existing data from the project schedules for each individual task to find optimal set of parameters for GA as Advanced Stochastic Time-Cost Tradeoff (ASTCT) method to solve TCTO problem. Ashuri \& Tavakolan (2015) considered the Pareto front optimization of resources along with the time and cost and presented a Shuffled Frog Leaping Algorithm. Pathak \& Srivastava (2015) developed a new technique for project planners for TCTO in fuzzy environment. Kaveh, Khanzadi, Alipour, \& Naraky (2015) showed that two new metaheuristic algorithms, Charged System Search (CSS) and Colliding Body Optimization (CBO), are utilized for solving this problem. The results show that both of these algorithms find reasonable solutions; however, $\mathrm{CBO}$ could find the result in a less computational time having a better quality. Shen, Hassani, \& Shi (2016) considered the problem with Coub-Douglas production function and hybrid GA. Hou, Zhao, Wu, Moon, \& Wang (2017) formulated a FA to target the optimal combination of the project makespan (start time, finish time) and execution mode of each project activity by using a series of unique mathematical models. Elloumi, Fortemps, \& Loukil (2017) developed a novel evolutionary algorithm for the problem under mode change disruption. Zheng \& Zhong (2017) presented hybrid GA for TCTO problem considering the environmental impact. He, He, Liu, \& Wang (2017) suggested Variable Neighbourhood Search and Tabu Search for TCTO problem to minimize the maximal cash flow gap.

On the other hand, the researchers who studied multi objective optimization problems have focused on the hybrid metaheuristic methods. Mehdizadeh, Hajipour, \& Mohammadizadeh (2015) investigated a multi-item capacitated lotsizing problem. They develop a bi-objective mathematical programming model with two conflicting objectives. They 
propose two novel Pareto-based multi-objective metaheuristic algorithms called Multi-objective Vibration Damping Optimization and the Non-dominated Ranking GA. Alaghebandha, Hajipour, \& Hemmati (2017) studied on the formulation of a sequencing problem with the dual goals of varying the parts utilization and varying the workload which are typically inversely correlated with each other, and therefore the simultaneous optimization of both is challenging. Owing to the NP-hardness of the problem, they introduce a discrete PSO algorithm, memetic algorithm, Weighted Sum Multi-objective GA and Non-dominated Sorting GA to solve problem where these objectives are to be optimized simultaneously.

\section{Metaheuristic Methods}

Methodology

The mathematical complexity of TСТО problem is directly affected by the size of the project network. In other words, the complexity of the problem grows exponentially as the number of the activities. Therefore the problem of TCTO is strongly NP-hard which means that TCTO cannot be solved with deterministic solution methods (Deineko \& Woeginger, 2001).

Recently, TCTO problems have been solved using metaheuristic methods. Metaheuristic methods can be useful and effective especially when a problem is difficult to solve with exact methods (Sörensen, Sevaux, \& Glover, 2018). They are also preferred frequently when the research field is large and complicated. Also, achieving the approximate results in a short time provides sufficient accuracy. These algorithms give quite reasonable solutions, but do not guarantee exactly optimum results (Yang, Deb, \& Fong, 2014).

Hybrid optimization technique is a successful combination of metaheuristic algorithm with another optimization algorithm that can display a more robust behavior and exhibit greater flexibility against complex and difficult problems (Blum, Aguilera, Roli, \& Sampels, 2008). In this study, integration of these two swarm intelligence metaheuristics is aimed for solving TCTO problem. The metaheuristics, PSO and FA have recently proved to be successful approaches to solve complex optimization problems using swarm intelligence. PSO is a powerful optimization technique for solving multimodal discrete optimization problems. Similarly, FA that imitates flashing behavior of fireflies is known to be efficient and robust for solution of combinatorial optimization problems (Shelokar, Siarry, Jayaraman, \& Kulkarni, 2007).

Recently, hybridizing local search strategies with PSO and FA has attracted more attentions, and the experimental results verify the promising comprehensive performance, including faster convergence speed and higher solution quality, of the hybrid algorithms (Li, Yang, \& Nguyen, 2012; Huang, Liu, Su, \& Yang, 2013). Meanwhile, detecting strategies (Xia, Liu, \& Hu, 2014) and opposition-based learning (OBL) strategies (Yu, Zhu, Ma, \& Mao, 2015) are also introduced to help the population to jump out of locally optimal solutions. Extensive experimental results manifest that the comprehensive performance of a hybrid algorithm can be dramatically improved if various merits within different algorithms/strategies are fully utilized by a proper integration mechanism (Xia, Gui, He, Xie, Wei, Xing, Wu, \& Tang, 2018).

The explanatory information about the characteristics and principles of FA and PSO is mentioned in the following subsections in order to make the novel hybrid method F-PSO more comprehensible.

\section{Firefly Algorithm (FA)}

FA is a relatively new nature-inspired optimization method that was first proposed by Yang (2010). As a stochastic metaheuristic algorithm, FA incorporates randomness into a search process (Fister, Yang, Brest, \& Fister, 2014). Even though FA is similar to PSO, it has proved to be much simpler in algorithm implementation (Kora \& Rama Krishna, 2016). On the other hand, the method has proved effective in solving multimodal optimization problems (Yang \& Wang, 2016).

According to FA process, some idealized conditions are adopted to search an optimal solution. These conditions are as follows:

- The fireflies are attracted by other fireflies according to their brightness and distance between them.

- The attractiveness between two firefly colonies is proportionally increased with the brightness.

- The brightness of a firefly is associated with objectives and if there is no firefly brighter than the other, that firefly will update.

In FA, it is assumed that there is a group of glowing fireflies. The attractiveness and the variation of the light intensity are the most important factors to be considered to formulate the behavior of the fireflies. Here, the light intensity $I$ is 
proportional to the objective function of the problem desired to be optimized. The brightness of a firefly depends on the intensity of light emitted by the firefly. The light intensity / varies with distance $r$ which is expressed by the following Eq. (7).

$$
I(r)=10 e^{-\gamma r^{2}}
$$

where 10 denotes the intensity of the light at the source, and $v$ is a fixed light absorption coefficient .The distance $r$ depends on the attractiveness which is calculated according to the Eq. (8).

$$
\beta(r)=\beta 0 e^{-\gamma r^{2}}
$$

where $\beta_{0}$ is the largest attraction. All fireflies have a unique attractiveness $\beta$, which indicates the ability to attract other fireflies. The attractiveness is related to the distance factor $r_{i j}$ at locations $s_{i}$ and $s_{j}$, and between the two corresponding fireflies, $i$ and $j$ are given by Eq. (9).

$$
r_{i j}=\left\|s_{i} s_{j}\right\|=\sqrt{\sum_{k=1}^{d}\left(s_{i k}-s_{j k}\right)^{2}}
$$

where $s_{i k}$ is the $\mathrm{k}$-th element of the $i^{\text {th }}$ firefly position within the search space. Each firefly $i$ move to another more attractive firefly $j$ and the movement is computed as in the Eq. (10).

$$
s_{j}=s_{i}+\beta_{0} e^{-\gamma r_{i j}^{2}}\left(s_{j}-s_{i}\right)+\alpha\left(\operatorname{rand}-\frac{1}{2}\right) \quad \alpha \in[0,1]
$$

Eq. (10) consists of three terms. The first term of the equation shows the position of the firefly $i$. The second term demonstrates to the attractiveness, while the third term depends on the randomized move of the firefly $i$ within the search space. This term consists of the randomized parameter $\alpha$, and randomly selected number between the interval $[0,1]$ as rand adopted from a Gaussian distribution. The process of FA is demonstrated on a flowchart in Figure 1.

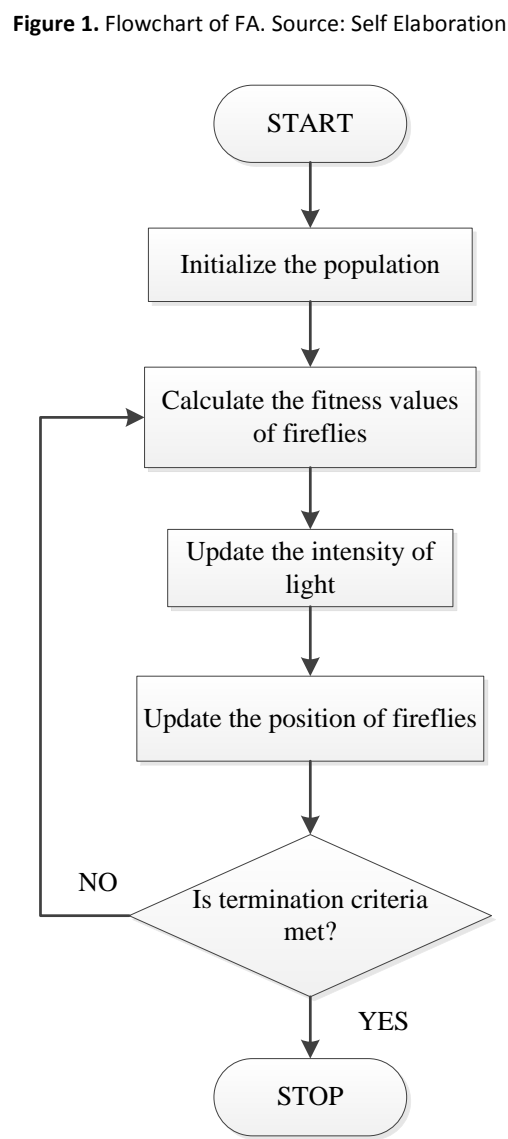




\section{Particle Swarm Optimization (PSO)}

Particle swarm optimization (PSO) is a type of swarm based algorithm inspired by nature. On the basis of such algorithms, living community called swarm has movements which are related to psychosocial factors. In PSO, each individual is called a particle, and the interactions of particles create the swarm intelligence. The particles of a community are better than the individual situation in terms of their observative and cognitive abilities. This status is particularly seen in bees, birds, fish and even bacteria. In behaviors such as hunting, migrating and escaping, each particle contributes to the consciousness of the swarm through its previous experiences and instincts. At this point, some particles lead the swarm, while the other particles follow the pioneers as a harmonious member of the swarm (Rajasekhar, Lynn, Das, \& Suganthan, 2017).

PSO was introduced by Kennedy \& Eberhart (1995) as a new optimization method in which each particle represents a possible solution.

According to process of PSO, if the position and velocity of the particle $i$ which is in the solution space at the moment of $t$ are shown $x_{i}(t)$ and $v_{i}(t)$ respectively, then the position of the particle $i$ at the moment of $(t+1)$ can be expressed as in the Eq. (11).

$$
x_{i}(t+1)=x_{i}(t)+v_{i}(t+1)
$$

The component which is shown as the velocity vector in the expression is one of the main elements of the system. The velocity vector includes information which is obtained by both its own and neighbor's experiences. In PSO method, the personal experiential knowledge is called cognitive component of the particle and the component obtained from neighbors is also known as the social component. The position of each particle which represents a solution is updated for every iteration in the algorithm. This update is made on the basis of each particle's best position lbest (best in the locally) and the best of the swarm $g_{\text {best }}$ (best in the globally) for each dimension $j \in 1, \ldots, N$, where $N$ is the dimension of the problem. For this reason, it is very important to define the velocity vector correctly, which has both cognitive and social components. Hence, $v_{i j}$ represents the $j^{\text {th }}$ element of the velocity vector of the $i^{\text {th }}$ particle. Thus, the velocity of particle $i$ is updated by Eq. (12)

$$
v_{i j}(t+1)=w v_{i j}(t)+c_{1} r_{1 j}(t)\left[l_{\text {best }}(t)-x_{i j}(t)\right]+c_{2} r_{2 j}(t)\left[g_{\text {best }}(t)-x_{i j}(t)\right]
$$

where $w$ is the inertia weight which varies 0.8 to $1.2, c_{1}$ and $c_{2} \sim U(0,2)$ are the acceleration coefficients, and also $r_{1}$, $r_{2} \sim U(0,1)$ are stochastic random numbers. The inertia weight $w$ influences the ability of the algorithm to search solutions locally or globally. If the value of $w$ decreases, then algorithm tends to search locally, but if $w$ increases, globally search is more possible. Selecting all the coefficients and parameters used in the PSO should be compatible with the structure of the problem. This issue is quite essential in terms of facilitating the access of optimum.

The personal best position of particle $i$ is the best point which is visited by particle $i$ so far. Actually best position results the best fitness value. Hereby the personal best of a particle at time $t$ is updated as Eq. (13) where $f$ denotes the objective function that has to be minimized.

$$
\begin{array}{ll}
y_{i}(t+1)=\quad y_{i}(t), & f\left(x_{i}(t+1)\right) \geq f\left(y_{i}(t)\right) \\
x_{i}(t+1), & f\left(x_{i}(t+1)\right)<f\left(y_{i}(t)\right)
\end{array}
$$

In the Eq. 13, $f$ function represents the fitness function. This function indicates that the position of a particle is close to optimum or not. Eq. 14 is used to obtain the global best position $\left(y_{g}\right)$ from the individual best position.

$$
y_{g}(t)=\min \left\{f\left(x_{i}(t)\right), \ldots, f\left(x_{n}(t)\right)\right\}
$$

A fundamental PSO algorithm is outlined in Figure 2. 


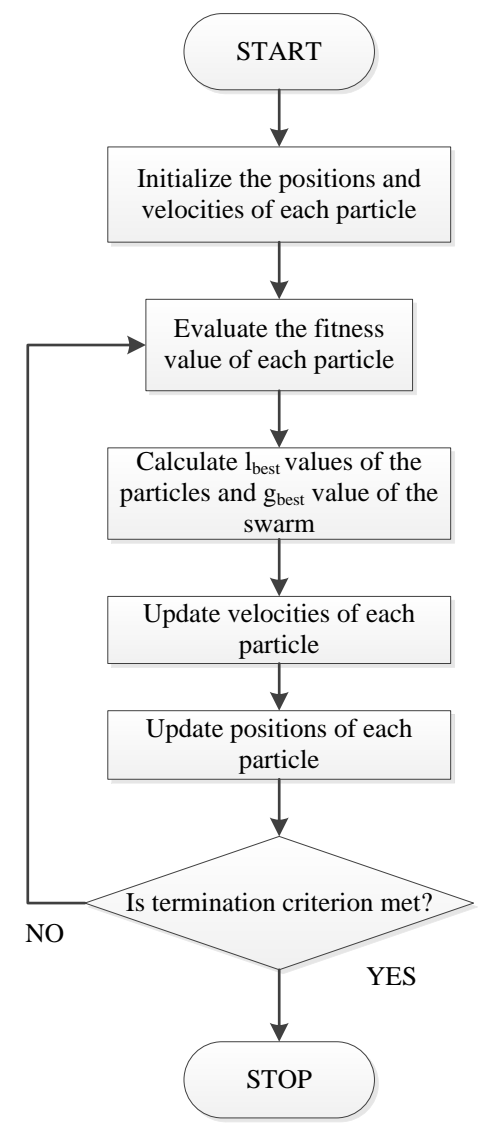

Novel hybrid algorithm Firefly-Particle Swarm Optimization (F-PSO)

Combining two or more metaheuristic algorithms means enhancing the advantages of the algorithms to create the hybrid algorithm that has practical value. Also the hybrid algorithm overcomes the shortcomings of the base algorithms and widens the application areas.

In this research, the novel hybrid metaheuristic method named firefly-particle swarm optimization (F-PSO) is introduced in order to solve TCTO problem. F-PSO is developed by modifying FA and PSO. Both algorithms have their own advantages and wide range application areas. The movement of an individual is only depends on all other brighter individuals in FA. Thus, the experimental knowledge of the individual can not affect the searching behavior. Therefore, while the brightest individual is located in a local optimum, the population is easily trapped into premature convergence. In contrast with FA, PSO takes into account historical knowledge of particles to guide them to search for promising regions. Owing to the hybridization of these well-studied metaheuristic methods, F-PSO is characterized by high speed and quick convergence properties. The specifications of F-PSO are described below in detail.

The algorithm of F-PSO which visualized in Figure 3 consists of two main phases. The algorithm implements FA in the first phase of the method, and then PSO is applied in the second phase. FA works to refine the randomized population. After first elimination, PSO handles the fireflies as particles to search the solution space and optimize the problem globally.

In the hybrid algorithm, the elimination rate is accepted as $80 \%$, after many experimental runs are performed (Albayrak, 2017). As a result of tests, this determined rate gives the most reasonable and consistent outputs. If this rate is increased to over $90 \%$, the elimination rate is not able to contribute to hybridization of the algorithms. Similarly, if the rate is reduced fewer than $75 \%$, the solution space affects negatively due to contraction. Consequently, the rate of elimination is recommended in the range of $80 \%-85 \%$ for this type of problems. The phases of the algorithm are explained below.

Phase 1. FA is used to obtain the elite population for PSO.

(1.a). Initialize the firefly population which has $n$ randomized individuals.

(1.b). Calculate the fitness values of each firefly by using objective function.

(1.c). Update the fireflies in according to light intensity and position. 
(1.d). Determine the top $0.8 n$ in ranked population.

(1.e). Forward the elite population to PSO algorithm.

Phase 2. PSO is used to search the global best individual of the population.

(2.a). Initialize PSO algorithm considering elite population.

(2.b). Calculate the fitness values of particles.

(2.c). Find the global best position in the population.

(2.d). Update the particles in according to positions and velocities.

(2.e). Check whether the termination criteria are met; if so, terminate the algorithm; otherwise, follow the step (1.b).

According to the algorithm flow, the fireflies explore larger search area initially. Thus, convergence speed of the algorithm increases and PSO process performs global exploration more efficiently. The F-PSO algorithm is simply structured and easy to use, while demonstrating great robustness and fast convergence in solving multi objective global optimization problem.

\section{Verification of F-PSO}

In order to test and verify F-PSO, there are two common benchmark functions that can be used. These functions provide a standardized comparison between PSO, FA, GA, ACO and F-PSO. Both reliability and accuracy abilities of the algorithms can be investigated and compared through benchmark functions which are suitable with discrete and multimodal optimization problems in the literature (Ma et al., 2014). Also the selected functions $f_{1}$ and $f_{2}$ are two of the most competitive benchmarks due to many steep hollows with multiple local optimum points. The function specifications are shown in Table 1.

Table 1. Specifications of benchmark functions. Source: Velásquez, 2010.

\begin{tabular}{lcll}
\hline Function name & \multicolumn{1}{c}{ Test function } & Variable domain & Global optimum \\
\hline Rastrigin (1974) & $f_{1}(x)=\sum_{i=1}^{D}\left(x_{i}^{2}-10 \cos \left(2 \pi x_{i}\right)+10\right)$ & $x_{i} \in[-5.12,5.12]$ & $x_{i}=0, f_{1}(x)=0$ \\
Griewank (1981) & $f_{2}(\mathrm{x})=\frac{1}{4000} \sum_{i=1}^{D} x_{i}^{2}-\prod_{i=1}^{D} \cos \left(\frac{x_{i}}{\sqrt{i}}\right)+1$ & $x_{i} \in[-5.12,5.12]$ & $x_{i}=0, f_{2}(x)=0$ \\
\hline
\end{tabular}

For the implementation of the benchmark functions, the experimental parameters of PSO, FA, GA, ACO and F-PSO are given in Table 2.

Table 2. Experimental parameters of the algorithms. Source: Self elaboration.

\begin{tabular}{|c|c|c|c|c|c|}
\hline $\begin{array}{c}\text { Exp. } \\
\text { parameters }\end{array}$ & PSO & $\mathrm{FA}$ & GA & $\mathrm{ACO}$ & F-PSO \\
\hline$w$ & 0.85 & - & - & - & 0.85 \\
\hline$c$ & 1.35 & - & - & - & 1.35 \\
\hline$r$ & 0.5 & - & - & - & 0.5 \\
\hline$V_{\max }$ & 0.5 & - & - & - & 0.5 \\
\hline$\alpha$ & - & 0.2 & - & - & 0.2 \\
\hline$B$ & - & 0.2 & - & - & 0.2 \\
\hline$\gamma$ & - & 0.9 & - & - & 0.9 \\
\hline$\delta$ & - & 0.95 & - & - & 0.95 \\
\hline $\begin{array}{l}\text { Number of } \\
\text { chromosome }\end{array}$ & - & - & 10 & - & - \\
\hline Crossover & - & - & $\begin{array}{l}\text { single point and } \\
\text { double point }\end{array}$ & - & - \\
\hline Mutation & - & - & single bit random & - & - \\
\hline$\rho$ & - & - & - & 0.8 & \\
\hline$\rho_{o}$ & - & - & - & 0.2 & - \\
\hline $\begin{array}{c}\text { Search space } \\
\text { limit }\end{array}$ & - & - & - & {$\left[\begin{array}{ll}-20 & 20\end{array}\right]$} & - \\
\hline Pop. size & 40 & 40 & 40 & 40 & 40 \\
\hline Max. iteration & 1000 & 1000 & 1000 & 1000 & 1000 \\
\hline
\end{tabular}


Searching for the minimum value of the functions, 40 experiments are carried out for each method which is limited 1000 iterations. The results are summarized in Table 3 included best, mean and standard deviation values. As can be seen from Tab. 3, the results show that F-PSO outperforms the results of standard algorithms. In other words, F-PSO is the best method in terms of solving the functions $f_{1}$ and $f_{2}$ which are highly multimodal.

The exploitation and exploration are two major factors among the many components of PSO and FA algorithms in order to search the solution space on a global scale efficiently. The enhanced searching ability of F-PSO is based on consolidation of powerful algorithms properly. The two-stage structure of the hybrid algorithm prevents to get stuck in local optimum which is the main shortcoming of the base algorithms. The prior knowledge obtained FA guides global search process for PSO stage. Hereby, better solution candidates are left to the secondary process.

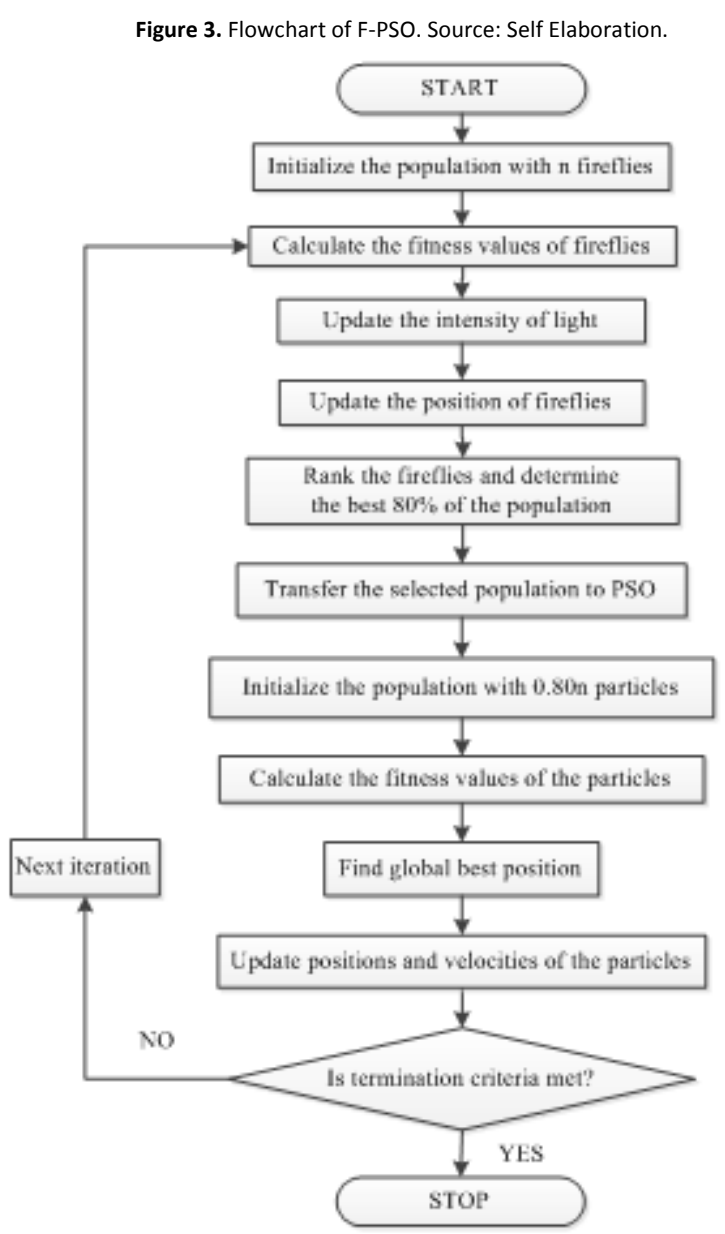

\begin{tabular}{llccc}
\multicolumn{5}{c}{ Table 3. Test results of the methods. Source: Self Elaboration. } \\
\hline Function name & Method & Best value & Mean value & Std. deviation \\
\hline Rastrigin & PSO & 0.8351 & 0.9835 & 0.1211 \\
& FA & 0.6433 & 0.8821 & 0.1953 \\
& GA & 0.7706 & 0.9145 & 0.1738 \\
GCO & 0.6179 & 0.7224 & 0.1244 \\
& F-PSO & 0.5211 & 0.6374 & 0.0936 \\
& PSO & 0.0228 & 0.0454 & 0.0185 \\
& FA & 0.0723 & 0.0889 & 0.0249 \\
& GA & 0.0812 & 0.1003 & 0.0209 \\
& ACO & 0.0798 & 0.0926 & 0.0227 \\
& F-PSO & 0.0197 & 0.0256 & 0.0168 \\
\hline
\end{tabular}

\section{Application of Time-Cost Trade-Off (TCTO)}

The application of F-PSO is examined on a sample project which includes TCTO problem. The sample project is selected from the literature that enables comparison. This 18-activity network and time-cost components were described in (Feng et al., 1997). Multiplying the number of execution modes with 18 activities by each other, there are approximately 5.9 billion combinations in the entire project. 
For this implementation, F-PSO procedures are coded MATLAB R2012a. Then, the algorithm is run on a personal computer configured with Intel Core2, 4 GB RAM, Windows10.

In order to observe the coherence of the solutions, the execution is repeated 20 times (Albayrak, 2017). Because the true Pareto front (i.e. non-dominated solutions) is difficult to be generated by a few run.

\section{Application Example}

The project is visualized with an activity-on-node (AON) network in Figure 4. Hereby, the precedence relationships can be seen in this figure easily. In sample project, each activity has different execution modes corresponding time and cost options. The activity numbers, predecessors, durations and cost alternatives are presented in Table 4. Along with five execution modes of construction, duration varies from faster to slower and similarly, cost varies from cheaper to more expensive. Additionally, the problem is considered to be minimized according to total project duration and cost.

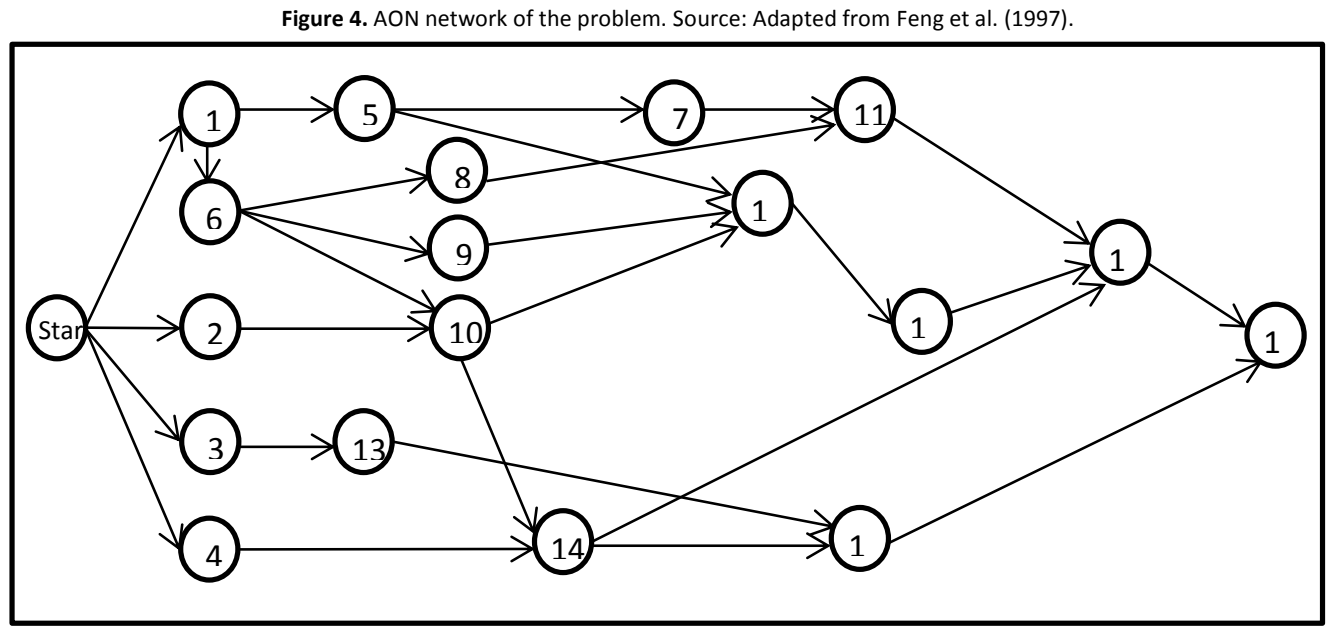

Table 4. 18-activity TCTO problem. Source: Feng et al. (1997)

\begin{tabular}{|c|c|c|c|c|c|c|c|c|c|c|c|}
\hline \multirow{2}{*}{$\begin{array}{l}\text { Act. } \\
\text { No. }\end{array}$} & \multirow{2}{*}{$\begin{array}{l}\text { Pred. } \\
\text { Activity }\end{array}$} & \multicolumn{2}{|l|}{ Mode 1} & \multicolumn{2}{|l|}{ Mode 2} & \multicolumn{2}{|l|}{ Mode 3} & \multicolumn{2}{|l|}{ Mode 4} & \multicolumn{2}{|l|}{ Mode 5} \\
\hline & & $\begin{array}{l}\text { Duration } \\
\text { (days) }\end{array}$ & $\begin{array}{l}\text { Cost } \\
(\$)\end{array}$ & $\begin{array}{l}\text { Duration } \\
\text { (days) }\end{array}$ & $\begin{array}{l}\text { Cost } \\
(\$)\end{array}$ & $\begin{array}{l}\text { Duration } \\
\text { (days) }\end{array}$ & $\begin{array}{l}\text { Cost } \\
(\$)\end{array}$ & $\begin{array}{l}\text { Duration } \\
\text { (days) }\end{array}$ & $\begin{array}{l}\text { Cost } \\
(\$)\end{array}$ & $\begin{array}{l}\text { Duration } \\
\text { (days) }\end{array}$ & $\begin{array}{l}\text { Cost } \\
(\$)\end{array}$ \\
\hline 1 & - & 14 & 2400 & 15 & 2150 & 16 & 1900 & 21 & 1500 & 24 & 1200 \\
\hline 2 & - & 15 & 3000 & 18 & 2400 & 20 & 1800 & 23 & 1500 & 25 & 1000 \\
\hline 3 & - & 15 & 4500 & 22 & 4000 & 33 & 3200 & - & - & - & - \\
\hline 4 & - & 12 & 45000 & 16 & 35000 & 20 & 30000 & - & - & - & - \\
\hline 5 & 1 & 22 & 20000 & 24 & 17500 & 28 & 15000 & 30 & 10000 & - & - \\
\hline 6 & 1 & 14 & 40000 & 18 & 32000 & 24 & 18000 & - & - & - & - \\
\hline 7 & 5 & 9 & 30000 & 15 & 24000 & 18 & 22000 & - & - & - & - \\
\hline 8 & 6 & 14 & 220 & 15 & 215 & 16 & 210 & 21 & 208 & 24 & 120 \\
\hline 9 & 6 & 15 & 300 & 18 & 240 & 20 & 180 & 23 & 150 & 25 & 100 \\
\hline 10 & 2,6 & 15 & 450 & 22 & 400 & 33 & 320 & - & - & - & - \\
\hline 11 & 7,8 & 12 & 450 & 16 & 350 & 20 & 300 & - & - & - & - \\
\hline 12 & $5,9,10$ & 22 & 2000 & 24 & 1750 & 28 & 1500 & 30 & 1000 & - & - \\
\hline 13 & 3 & 14 & 4000 & 18 & 3200 & 24 & 1800 & - & - & - & - \\
\hline 14 & 4,10 & 9 & 3000 & 15 & 2400 & 18 & 2200 & - & - & - & - \\
\hline 15 & 12 & 12 & 4500 & 16 & 3500 & - & - & - & - & - & - \\
\hline 16 & 13,14 & 20 & 3000 & 22 & 2000 & 24 & 1750 & 28 & 1500 & 30 & 1000 \\
\hline 17 & $11,14,15$ & 14 & 4000 & 18 & 3200 & 24 & 1800 & - & - & - & - \\
\hline 18 & 16,17 & 9 & 3000 & 15 & 2400 & 18 & 2200 & - & - & - & - \\
\hline
\end{tabular}

Albayrak (2017) performed many preliminary experiments and suggested the number of iterations as 500 . Similarly, the population size was proposed as 20 for this problem. These suggestions are adopted for this paper. To perform the problem with F-PSO, the parameters of the algorithm are set as Table 5. 


\begin{tabular}{|c|c|}
\hline \multicolumn{2}{|c|}{ Parameters of F-PSO } \\
\hline$w$ & 0.85 \\
\hline$c$ & 1.35 \\
\hline$v_{\max }$ & 0.5 \\
\hline$\alpha$ & 0.2 \\
\hline B & 0.2 \\
\hline$\gamma$ & 0.9 \\
\hline$\delta$ & 0.95 \\
\hline Pop. size & 20 \\
\hline Max. iteration & 500 \\
\hline
\end{tabular}

The FA algorithm starts with random generation of the initial population. Each obtained value is represented by a firefly which is a candidate of the optimum result. The light intense of fireflies indicates the fitness value according to the objective function. Then fireflies are compared with each other and the best $80 \%$ of the population is transferred to PSO process. The position and velocity update operators are applied to population repeatedly until the termination condition is satisfied. The best global optimal value is provided by the end of the procedure.

The method is able to search the solution space and archive the dominated and non-dominated solutions in the successive iterations of the algorithm. After the analysis, the dominated solutions are eliminated and non-dominated solutions are kept. This leads to separate 24 Pareto optimal solutions for this project. Each of these solutions correspond an optimal trade-off point between total time and cost. Pareto front solutions are summarized in Table 6 and Figure 5 shows the curve of the time-cost trade-off relating to this project, where the horizontal axis represents total duration and the vertical axis represents project cost. In according to ТСТО curve, the project duration varies from 100 to 152 days and the cost values vary from $\$ 134.870,00$ to $\$ 100.240,00$ totally. Each of Pareto solutions represents an optimal trade-off for the activities of the project entirely.

Table 6. Pareto solutions of TCTO. Source: Self Elaboration.

\begin{tabular}{|c|c|c|c|c|c|}
\hline Solution No. & Duration (days) & Cost $\left(\$ \times 10^{3}\right)$ & Solution No. & Duration (days) & Cost $\left(\$ \times 10^{3}\right)$ \\
\hline 1 & 100 & 134.87 & 13 & 122 & 104.02 \\
\hline 2 & 103 & 129.8 & 14 & 123 & 103.85 \\
\hline 3 & 104 & 126.9 & 15 & 124 & 102.7 \\
\hline 4 & 105 & 121.12 & 16 & 125 & 102.1 \\
\hline 5 & 108 & 116.415 & 17 & 126 & 101.87 \\
\hline 6 & 111 & 114.09 & 18 & 128 & 101.05 \\
\hline 7 & 113 & 111.915 & 19 & 131 & 100.98 \\
\hline 8 & 115 & 109.9 & 20 & 135 & 100.75 \\
\hline 9 & 118 & 107.52 & 21 & 139 & 100.66 \\
\hline 10 & 119 & 106.27 & 22 & 143 & 100.52 \\
\hline 11 & 120 & 104.92 & 23 & 147 & 100.378 \\
\hline 12 & 121 & 104.77 & 24 & 152 & 100.24 \\
\hline
\end{tabular}

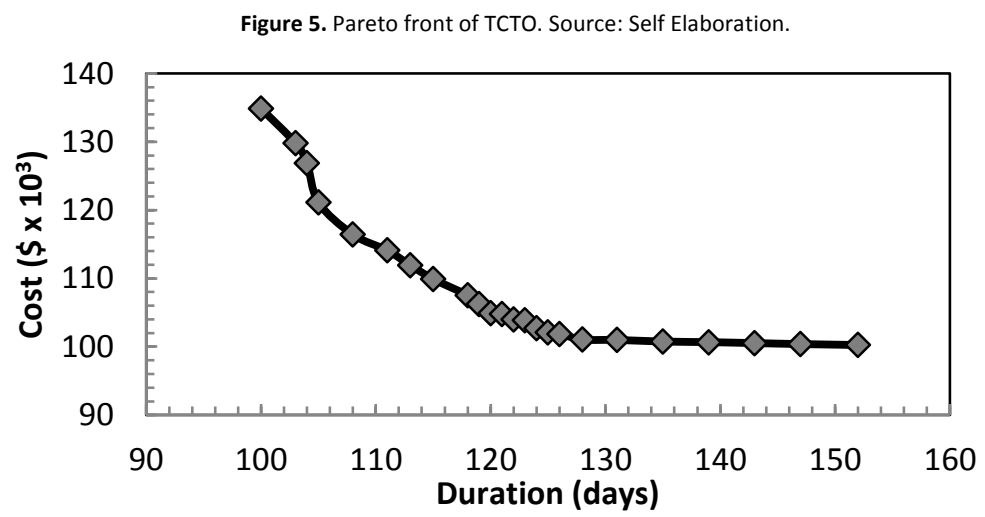


The Pareto front generated by the proposed model provides critical information needed by decision makers to create optimal designs under project time and cost constraints. The proposed model allows decision makers to make timely, informed decisions based on time and cost preferences.

In F-PSO, which is limited to 500 iterations, the values forming the Pareto front were observed in the first 200 iterations. The graphic of number of iterations-fitness value is given in the Figure 6 . According to the Figure 6, it is concluded that the selected parameters and the number of iterations are sufficient.

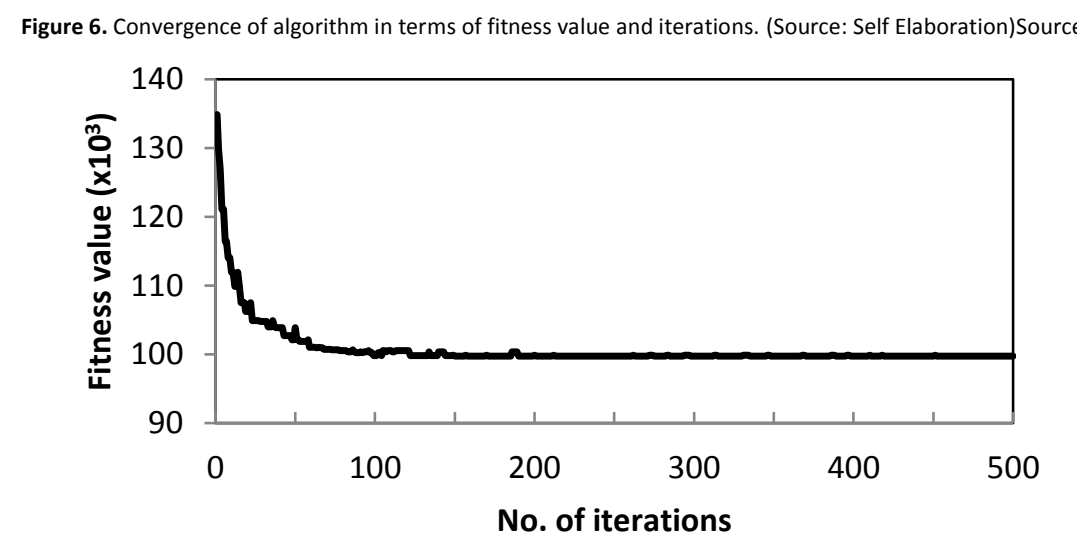

In order to validate the results, Elbeltagi et al. (2005), Geem (2010) and Lee et al. (2015) are considered in the literature because 18-activity project is examined by them using other metaheuristic algorithms. The algorithms to be compared are as follows: Particle Swarm Optimization (PSO), Ant Colony Optimization (ACO), Genetic Algorithm (GA), Memetic Algorithm (MA), Harmony Search (HS) and Advanced Stochastic Time-Cost Tradeoff (ASTCT). The comparison confirms that the current model is capable of obtaining better solution in terms of both cost and duration results. According to comparison, harmony search optimal values are second optimal among seven metaheuristic algorithms. The results are compared visually as shown in Table 7.

Table 7. Comparison of seven metaheuristic algorithm results on the same problem. Source: Self Elaboration.

\begin{tabular}{|c|c|c|c|c|c|c|c|}
\hline & \multicolumn{4}{|c|}{ (Elbeltagi et al., 2005) } & \multirow{2}{*}{$\frac{(\text { Geem, 2010) }}{\mathrm{HS}}$} & \multirow{2}{*}{$\frac{(\text { Lee et al., 2015) }}{\text { ASTCT }}$} & \multirow{2}{*}{$\begin{array}{c}\text { This study } \\
\text { F-PSO }\end{array}$} \\
\hline & PSO & $\mathrm{ACO}$ & GA & $\mathrm{MA}$ & & & \\
\hline Min. C & 106270 & 106270 & 105770 & 106270 & 102320 & 106270 & 100240 \\
\hline Min. Dur.(days) & 110 & 110 & 113 & 110 & 105 & 110 & 100 \\
\hline
\end{tabular}

\section{Conclusions}

Every construction project has its own unique conditions. The uniqueness of construction projects also means that the external influences and constraints would be different, yet subject to change throughout the project timeline. Therefore, it is important to attain time-cost alternatives that are appropriate to the project conditions. This approach can be achieved through metaheuristics and Pareto front.

This paper presents a novel method named F-PSO for solving TCTO problem which is one of the most difficult discrete optimization problems in construction project planning, due to various variables and constraints. F-PSO is based on the hybridization of FA and PSO to improve the exploration and exploitation abilities of both algorithms. Also, better optimality of the solutions and quick convergence are aimed when building the hybrid method. For this purpose, the poor solutions according to fitness values are disqualified in FA section of the process, before passing PSO that is the second step of the algorithm. Primarily, two benchmark equations are tested to verify the algorithm. After verification, F-PSO is implemented on a well-known 18-activity TCTO problem which enables the comparison. The proposed algorithm finds out the optimal solution and defines the Pareto front. The results are compared to other five metaheuristic algorithms which are applied on the same problem. The comparison shows that, F-PSO is generally found to perform better than other algorithms in terms of both minimum cost and duration results. The improvement of the algorithm combination provides an efficient method regarding to obtain shorter and more economical alternatives of the construction projects. 
The proposed F-PSO is simple, robust and efficient. It does not impose any limitation on the number of objectives and can be extended to include more objectives. Further minor modifications of F-PSO algorithm hold potential to resolve other multi-objective optimization problems in the field of construction management such as the tradeoffs among performance, cost, and reliability; time, cost and safety tradeoffs; and resource-constrained and resource-leveling in project scheduling activities.

In this study, the quality and risk factors are not considered and they are assumed stable against the changes of time and cost. The idea of integrating such factors into study will provide new insights for future works.

Acknowledgement

This paper is based on the first author's PhD dissertation entitled "A Comparative Selection of Hybrid-Metaheuristic Methods on Time-Cost Trade-Off in Resource Constrained Construction Projects".

The authors would like to express their sincere thanks to the anonymous reviewers for valuable comments and suggestions on this paper.

Alaghebandha, M., Hajipour, V., \& Hemmati, M. (2017). Optimizing multi-objective sequencing problem in mixed-model assembly line on just-in-time: particle swarm optimization algorithm. International Journal of Management Science and Engineering Management, 12(4), $288-298$. https://doi.org/10.1080/17509653.2016.1258593

Albayrak, G. (2017). A comparative selection of hybrid-metaheuristic methods on time-cost trade-off in resource constrained construction projects. PhD Dissertation, Eskisehir Osmangazi University, Graduate School of Natural and Applied Sciences, Turkey.

Ashuri, B., \& Tavakolan, M. (2015). Shuffled Frog-Leaping Model for Solving Time-Cost-Resource Optimization Problems in Construction Project Planning. Journal of Computing in Civil Engineering, 29(1), 04014026. https://doi.org/10.1061/(ASCE)CP.1943-5487.0000315

Blum, C., Belsa Aguilera, M. J., Roli, A., \& Sampels, M. (2013). Hybrid Metaheuristics. Computational Intelligence (Vol. 7919). https://doi.org/10.1007/978-3-642-38516-2

Chen, S. P., \& Tsai, M. J. (2011). Time-cost trade-off analysis of project networks in fuzzy environments. European Journal of Operational Research, 212(2), 386-397. https://doi.org/10.1016/j.ejor.2011.02.002

Cheng, M. Y., \& Tran, D. H. (2014). Two-phase differential evolution for the multiobjective optimization of time-cost tradeoffs in resource-constrained construction projects. IEEE Transactions on Engineering Management, 61(3), 450-461. https://doi.org/10.1109/TEM.2014.2327512

De, P., Dunne, E. J., Ghosh, J. B., \& Wells, C. E. (1997). Complexity of the Discrete Time-Cost Tradeoff Problem for Project Networks. Operations Research, 45(2), 302-306. https://doi.org/10.1287/opre.45.2.302

Deǐneko, V. G., \& Woeginger, G. J. (2001). Hardness of approximation of the discrete time-cost tradeoff problem. Operations Research Letters, 29(5), 207-210. https://doi.org/10.1016/S0167-6377(01)00102-X

Elbeltagi, E., Hegazy, T., \& Grierson, D. (2005). Comparison among five evolutionary-based optimization algorithms. Advanced Engineering Informatics, 19(1), 43-53. https://doi.org/10.1016/j.aei.2005.01.004

Elloumi, S., Fortemps, P., \& Loukil, T. (2017). Multi-objective algorithms to multi-mode resource-constrained projects under mode change disruption. Computers and Industrial Engineering, 106, 161-173. https://doi.org/10.1016/j.cie.2017.01.029

Elmaghraby, S. E., \& Salem, A. (1981). Optimal linear approximation in project compression. Operations Research Technical Report, 171, North Carolina State University at Raleigh.

Eshtehardian, E., Afshar, A., \& Abbasnia, R. (2009). Fuzzy-based MOGA approach to stochastic time-cost trade-off problem. Automation in Construction, 18(5), 692-701. https://doi.org/10.1016/j.autcon.2009.02.001

Fan, K., You, W., \& Li, Y. (2013). An effective modified binary particle swarm optimization (mBPSO) algorithm for multi-objective resource allocation problem (MORAP). Applied Mathematics and Computation, 221, 257-267. https://doi.org/10.1016/j.amc.2013.06.039

Feng, C.-W., Liu, L., \& Burns, S. A. (1997). Using Genetic Algorithms to Solve Construction Time-Cost Trade-Off Problems. Journal of Computing in Civil Engineering, 11(3), 184-189. https://doi.org/10.1061/(ASCE)0887-3801(1997)11:3(184)

Feng, C.-W., Liu, L., \& Burns, S. A. (2000). Stochastic Construction Time-Cost Trade-Off Analysis. Journal of Computing in Civil Engineering, 14(2), 117126. https://doi.org/10.1061/(ASCE)0887-3801(2000)14:2(117)

Fister, I., Yang, X. S., Brest, J., \& Fister, I. Jr. (2014). On the randomized firefly algorithm. Cuckoo Search and Firefly Algorithm. Springer International Publishing, 27-48.

Fulkerson, D. R. (1961). A Network Flow Computation for Project Cost Curves. Management Science, 7(2), $167-178$. https://doi.org/10.1287/mnsc.7.2.167 
Geem, Z. W. (2010). Multiobjective Optimization of Time-Cost Trade-Off Using Harmony Search. Journal of Construction Engineering and Management, 136(6), 711-716. https://doi.org/10.1061/(ASCE)CO.1943-7862.0000167

Griewank, A. O. (1981). Generalized descent for global optimization. Journal of Optimization Theory and Applications, 34(1), 11-39. https://doi.org/10.1007/BF00933356

He, Z., He, H., Liu, R., \& Wang, N. (2017). Variable neighbourhood search and tabu search for a discrete time/cost trade-off problem to minimize the maximal cash flow gap. Computers and Operations Research, 78, 564-577. https://doi.org/10.1016/j.cor.2016.07.013

Hegazy, T. (1999). Optimization of construction time-cost trade-off analysis using genetic algorithms. Canadian Journal of Civil Engineering, 26(6), 685-697. https://doi.org/10.1139/199-031

Hendrickson, C., \& Au, T. (1989). Project management for construction. Prentice Hall, Englewood Cliffs, N.J., USA.

Hou, L., Zhao, C., Wu, C., Moon, S., \& Wang, X. (2017). Discrete Firefly Algorithm for Scaffolding Construction Scheduling. Journal of Computing in Civil Engineering, 31(3), 04016064. https://doi.org/10.1061/(ASCE)CP.1943-5487.0000639

Huang, S. J., Liu, X. Z., Su, W. F., \& Yang, S. H. (2013). Application of hybrid firefly algorithm for sheath loss reduction of underground transmission systems. IEEE Transactions on Power Delivery, 28(4), 2085-2092. https://doi.org/10.1109/TPWRD.2013.2265913

Huang, Y. S., Deng, J. J., \& Zhang, Y. Y. (2008). TI time-cost-quality tradeoff optimization in construction project based on modified ant colony algorithm. In Proceedings of the 7th International Conference on Machine Learning and Cybernetics, ICMLC (Vol. 2, pp. 1031-1035). https://doi.org/10.1109/ICMLC.2008.4620556

Kandil, A., \& El-Rayes, K. (2005). Parallel Computing Framework for Optimizing Construction Planning in Large-Scale Projects. Journal of Computing in Civil Engineering, 19(3), 304-312. https://doi.org/10.1061/(ASCE)0887-3801(2005)19:3(304)

Kaveh, A., Khanzadi, M., Alipour, M., \& Naraky, M. R. (2015). CBO and CSS algorithms for resource allocation and time-cost trade-off. Periodica Polytechnica Civil Engineering, 59(3), 361-371. https://doi.org/10.3311/PPci.7788

Kelley, J. E. (1961). Critical-Path Planning and Scheduling: Mathematical Basis. Operations Research, 9(3), 296-320. https://doi.org/10.1287/opre.9.3.296

Kennedy, J., \& Eberhart, R. (1995). Particle swarm optimization. Proceedings of IEEE International Conference on Neural Networks, $1942-1948$. https://doi.org/10.1109/ICNN.1995.488968

Klerides, E., \& Hadjiconstantinou, E. (2015). The stochastic discrete time-cost tradeoff problem with decision-dependent uncertainty. In Handbook on Project Management and Scheduling Vol. 2 (pp. 781-809). https://doi.org/10.1007/978-3-319-05915-0_6

Kora, P., \& Rama Krishna, K. S. (2016). Hybrid firefly and Particle Swarm Optimization algorithm for the detection of Bundle Branch Block. International Journal of the Cardiovascular Academy, 2(1), 44-48. https://doi.org/10.1016/j.ijcac.2015.12.001

Lee, H. G., Yi, C. Y., Lee, D. E., \& Arditi, D. (2015). An Advanced Stochastic Time-Cost Tradeoff Analysis Based on a CPM-Guided Genetic Algorithm. Computer-Aided Civil and Infrastructure Engineering, 30(10), 824-842. https://doi.org/10.1111/mice.12148

Leu, B. S., \& Yang, C. (1999). GA-Based Multicriteria Optimal for Construction Scheduling. Journal of Construction Engineering and Management, 125(December), 420-427. https://doi.org/10.1061/(ASCE)0733-9364(1999)125:6(420)

Li, C., Yang, S., \& Nguyen, T. T. (2012). A self-learning particle swarm optimizer for global optimization problems. IEEE Transactions on Systems, Man, and Cybernetics, Part B: Cybernetics, 42(3), 627-646. https://doi.org/10.1109/TSMCB.2011.2171946

Li, H., \& Love, P. (1997). Using Improved Genetic Algorithms to Facilitate Time-Cost Optimization. Journal of Construction Engineering and Management, 123(3), 233-237. https://doi.org/10.1061/(ASCE)0733-9364(1997)123:3(233)

Ma, L., Hu, K., Zhu, Y., Niu, B., Chen, H., \& He, M. (2014). Discrete and continuous optimization based on hierarchical artificial bee colony optimizer. Journal of Applied Mathematics, 2014 Article ID 402616, 20 pages. https://doi.org/10.1155/2014/402616

Mehdizadeh, E., Hajipour, V., \& Mohammadizadeh, M. R. (2015). A bi-objective multi-item capacitated lot-sizing model: Two pareto-based metaheuristic algorithms. International Journal of Management Science and Engineering Management, 11(4). https://doi.org/10.1080/17509653.2015.1086965

Mokhtari, H., Baradaran Kazemzadeh, R., \& Salmasnia, A. (2011). Time-cost tradeoff analysis in project management: An ant system approach. IEEE Transactions on Engineering Management, 58(1), 36-43. https://doi.org/10.1109/TEM.2010.2058859

Ng, S. T., \& Zhang, Y. (2008). Optimizing Construction Time and Cost Using Ant Colony Optimization Approach. Journal of Construction Engineering and Management, 134(September), 721-728. https://doi.org/10.1061/(ASCE)0733-9364(2008)134:9(721)

Pathak, B. K., \& Srivastava, S. (2015). Effects of project uncertainties on nonlinear time-cost tradeoff profile. Iranian Journal of Fuzzy Systems, 12(4), 79-100.

Pareto, V. (1906). Manuale di economia politica, societa editrice libraria. Milano, Italy. Translated into English by Schwier, A.S. (1971) Manual of political economy, New York: Macmillan.

Praščević, N. \& Praščević, Ž. (2014). Application of particle swarms for project time-cost optimization, GRAĐEVINAR, 66(12), 1097-1107, doi: https://doi.org/10.14256/JCE.1048.2014

Rajasekhar, A., Lynn, N., Das, S., \& Suganthan, P. N. (2017). Computing with the collective intelligence of honey bees - A survey. Swarm and Evolutionary Computation, 32, 25-48. https://doi.org/10.1016/j.swevo.2016.06.001 
Rastrigin, L.A. (1974). Extremal Control Systems. In Theoretical Foundations of Engineering Cybernetics Series, Moscow.

Salem, A. M., \& Elmaghraby, S. E. (1994). Optimal linear approximation in project compression. IIE Transactions (Institute of Industrial Engineers), 16(4), 339-347. https://doi.org/10.1080/07408178408975253

Shelokar, P. S., Siarry, P., Jayaraman, V. K., \& Kulkarni, B. D. (2007). Particle swarm and ant colony algorithms hybridized for improved continuous optimization. Applied Mathematics and Computation, 188(1), 129-142. https://doi.org/10.1016/j.amc.2006.09.098

Shen, Z., Hassani, A., \& Shi, Q. (2016). Multi-objective time-cost optimization using Cobb-Douglas production function and hybrid genetic algorithm. Journal of Civil Engineering and Management, 22(2), 187-198. https://doi.org/10.3846/13923730.2014.897966

Siemens, N. (1971). A Simple CPM Time-Cost Tradeoff Algorithm. Management Science, 17(6), B-354-B-363. https://doi.org/10.1287/mnsc.17.6.B354

Sonmez, R., \& Bettemir, Ö. H. (2012). A hybrid genetic algorithm for the discrete time-cost trade-off problem. Expert Systems with Applications, 39(13), 11428-11434. https://doi.org/10.1016/j.eswa.2012.04.019

Sörensen K., Sevaux M., \& Glover F. (2018). A history of metaheuristics. In: Martí R., Panos P., Resende M. (eds) Handbook of Heuristics. Springer, Cham. https://doi.org/10.1007/978-3-319-07153-4_4-1

Tareghian, H. R., \& Taheri, H. (2006). An application of randomized minimum cut to the project time/cost tradeoff problem. Applied Mathematics and Computation, 173(2), 1200-1207. https://doi.org/10.1016/j.amc.2005.04.063

Tareghian, H. R., \& Taheri, S. H. (2007). A solution procedure for the discrete time, cost and quality tradeoff problem using electromagnetic scatter search. Applied Mathematics and Computation, 190(2), 1136-1145. https://doi.org/10.1016/j.amc.2007.01.100

Vanhoucke, M., \& Debels, D. (2007). The discrete time/cost trade-off problem: Extensions and heuristic procedures. Journal of Scheduling, 10(4-5), 311-326. https://doi.org/10.1007/s10951-007-0031-y

Velásquez, J. D. (2010). An Enhanced Hybrid Chaotic Algorithm using Cyclic Coordinate Search and Gradient Techniques. Revista de Ingeniería, Universidad de Los Andes, 32, 45-53.

Xia, X., Gui, L., He, G., Xie, C., Wei, B., Xing, Y., ... Tang, Y. (2018). A hybrid optimizer based on firefly algorithm and particle swarm optimization algorithm. Journal of Computational Science, 26, 488-500. https://doi.org/10.1016/j.jocs.2017.07.009

Xia, X., Liu, J., \& Hu, Z. (2014). An improved particle swarm optimizer based on tabu detecting and local learning strategy in a shrunk search space. Applied Soft Computing Journal, 23, 76-90. https://doi.org/10.1016/j.asoc.2014.06.012

Yang, X. S. (2010). Firefly algorithm, stochastic test functions and design optimisation, International Journal of Bio-Inspired Computation, 2(2), 78-84.

Yang, X. S., Deb, S., \& Fong, S. (2014). Metaheuristic algorithms: Optimal balance of intensification and diversification. Applied Mathematics and Information Sciences, 8(3), 977-983. https://doi.org/10.12785/amis/080306

Yang, Z., \& Wang, J. (2016). Multistep wind speed forecasting using a novel model hybridizing singular spectrum analysis, modified intelligent optimization, and rolling elman neural network. Mathematical Problems in Engineering, 2016, Article ID 3623412, 21 pages. https://doi.org/10.1155/2016/3623412

Yu, S., Zhu, S., Ma, Y., \& Mao, D. (2015). Enhancing firefly algorithm using generalized opposition-based learning. Computing, 97(7), 741-754. https://doi.org/10.1007/s00607-015-0456-7

Zheng, H., \& Zhong, L. (2017). Discrete time-cost-environment trade-off problem and its application to a large-scale construction project. Proceedings of the Tenth International Conference on Management Science and Engineering Management (pp.1375-1382). https://doi.org/10.1007/978981-10-1837-4 111 